\title{
Space-charge effects in high brightness electron beam emittance measurements
}

\author{
S. G. Anderson and J. B. Rosenzweig \\ UCLA Department of Physics and Astronomy, 405 Hilgard Avenue, Los Angeles, California 90095
}

G. P. LeSage and J. K. Crane

Lawrence Livermore National Laboratory, Livermore, California 94550

(Received 21 September 2001; published 10 January 2002)

\begin{abstract}
The measurement of emittance in space-charge dominated, high brightness beam systems is investigated from conceptual, computational, and experimental viewpoints. As the self-field-induced collective motion in the low energy, high brightness beams emitted from photoinjector rf guns are more important in determining the macroscopic beam evolution than thermal spreads in transverse velocity; traditional methods for phase space diagnosis fail in these systems. We discuss the role of space charge forces in a traditional measurement of transverse emittance, the quadrupole scan. The mitigation of these effects by use of multislit- or pepper-pot-based techniques is explained. The results of a direct experimental comparison between quadrupole scanning and slit-based determination of the emittance of a $5 \mathrm{MeV}$ high brightness electron beam are presented. These data are interpreted with the aid of both envelope and multiparticle simulation codes. It is shown that the ratio of the beam's $\beta$ function to its transverse plasma wavelength plays a central role in the quadrupole scan results. Methods of determining the presence of systematic errors in quadrupole scan data are discussed.
\end{abstract}

DOI: $10.1103 /$ PhysRevSTAB.5.014201

PACS numbers: 05.45.-a, 29.27.Bd, 41.75.Ht, 41.85.Ja

\section{INTRODUCTION}

There exists an emerging class of very high brightness electron beams, which are created through photoemission from cathodes embedded in high field radio frequency guns, and driven by picosecond lasers. These systems, termed rf photoinjectors [1,2], produce high brightness beams by creating high charge beams with the time structure of the driving laser, and preserving both the temporal extent and the transverse phase space quality, despite the presence of extremely large space-charge forces. The preservation of the electron beam pulse length is accomplished by application of large amplitude rf accelerating fields, while the transverse phase space quality is controlled and optimized by judicious balancing of space charge defocusing and externally applied focusing forces. Perhaps one of the more remarkable aspects of the beam's evolution is that the transverse emittance can be greatly expanded and subsequently diminished during one plasma oscillation occurring under optimized beam transport conditions. This process, termed space charge emittance compensation, has been studied in detail in theoretical, computational, and experimental investigations [3-6].

As a result of the advancement in the understanding of the emittance compensation process in these beams, new photoinjectors are designed specifically to use external focusing and acceleration to balance the extreme spacecharge forces in the bunch [7]. The same care must be taken in the implementation of diagnostics for photoinjector beams. In particular, measurement schemes that deduce a beam's properties from its behavior in a drift region need to take into account the space-charge forces in the beam, as these forces usually control the beam's behavior. In addition, space charge can change the property one is attempting to measure in the region of measurement. For example, the emittance compensation process relies on the fact that the emittance changes (oscillates) in a drift region after a solenoid. Another issue in the measurement of photoinjector beams is the stability of the photocathode drive laser. Fluctuations in the energy and size of the drive laser cause shot-to-shot fluctuations in the beam size and emittance both directly and indirectly (by changing the space-charge forces and the emittance compensation process.) For this reason, in photoinjectors single shot measurements are preferable to those requiring many shots.

In the remainder of this paper, we review and examine the effects of space charge in two different emittance measurement techniques - the multislit-based and quadrupole (quad) scan systems. This paper builds on previous work on the subject of emittance measurement systems [8] and measuring the emittance of photoinjector beams [9]. We discuss here some of the relevant issues in Refs. [8] and [9] and clarify some key differences between this work and those. We then describe an experiment performed at the LLNL Thomson source photoinjector where these two techniques were used to measure the emittance of a highly space-charge dominated beam. The results of this experiment, which show that the emittance found with the quadrupole scan is in disagreement with that measured with the multislits (the quad scan results are consistently higher), are compared with simulation and analytical models. 


\section{MULTISLIT-BASED EMITTANCE MEASUREMENT}

The first purpose of a phase space measurement system based on multislit collimation [10] is to slice up the beam into physically separated linelike sources, or beamlets, as shown in Fig. 1. This slicing combined with a drift, which reveals the spread in velocities as spatial information at an intensity-sensitive detector, allows a full reconstruction of one of the beam's transverse phase planes, e.g., $\left(x, p_{x}\right)$. This technique is especially useful when implemented as a single-shot diagnostic in photoinjectors, where shot-byshot beam parameter fluctuations may dominate multishot measurements. Multishot variants of the slit-based method involving one or two slits, as well as the pepper-pot method (the 2D analog of the multislit system, which can give the four-dimensional transverse phase space), are discussed in detail in Ref. [8].

The method of determining the phase space from the multislit technique is reviewed below. After introduction of the technique, we discuss the use of the multislit array to mitigate collective effects in space-charge dominated beams which can be large enough to invalidate the measurement. While these effects have been mentioned in previous analyses [8-10], in this paper they are the focus of our concern. They are therefore examined in depth, not only in the context of the multislit method, but also in the quadrupole scan measurement.

The beamlets are created by the multislit mask at a constant spacing $w$, which is much larger than the slit width $d$. The beamlet distribution is then detected downstream of the multislit mask, and the beamlets resolved. The width of each beamlet gives a measure of the width of the transverse momentum distribution at each slit, and the centroid of the beamlets gives the correlated offset of the momentum distribution at each slit. Assuming a drift length $L$ between the multislit mask and the detecting plane, the measured trace space distribution is approximated by a series $(m=1, \ldots, N)$ of distinct angular distributions at regularly spaced spatial (we take this dimension to be $x$ ) intervals. The beamlets yield the correlated beam divergences

$$
x_{m, c}^{\prime}=\left\langle x_{m}-x_{m, c}\right\rangle / L=\left\langle x_{m}-m w\right\rangle / L,
$$

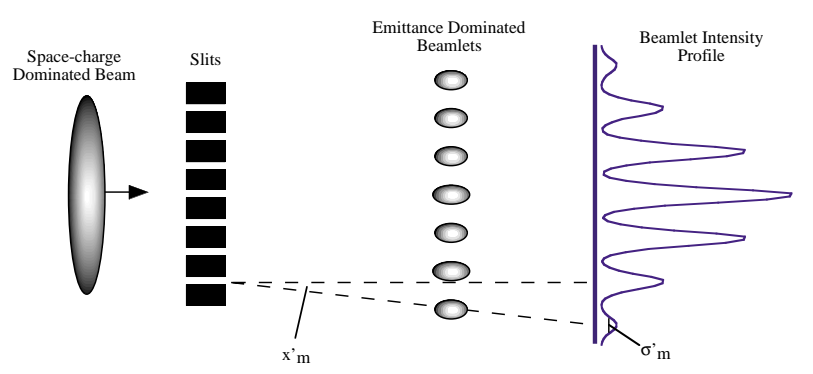

FIG. 1. (Color) Illustration of the multislit-based emittance measurement scheme. and the rms spreads in divergence

$$
\sigma_{m}^{\prime}=\sqrt{\left\langle x_{m}^{2}\right\rangle / L^{2}-\left(x_{m, c}^{\prime}\right)^{2}},
$$

where the average \langle\rangle is performed over the distribution in the $m$ th beamlet. Here we have assumed that the final spread in detected beamlet size is much greater than the slit width. Once these parameters are extracted from the data, a graphical trace space distribution can be constructed, as illustrated in Fig. 2.

Note that the trace space distribution is centered in both $x$ and $x^{\prime}$, by subtracting off the overall value of the centroids,

$$
x_{m, c}=\left.\left(x_{m, c}-x_{c}\right)\right|_{\mathrm{old}}=m w-\frac{\sum_{m=1}^{N} I_{m} m w}{\sum_{m=1}^{N} I_{m}},
$$
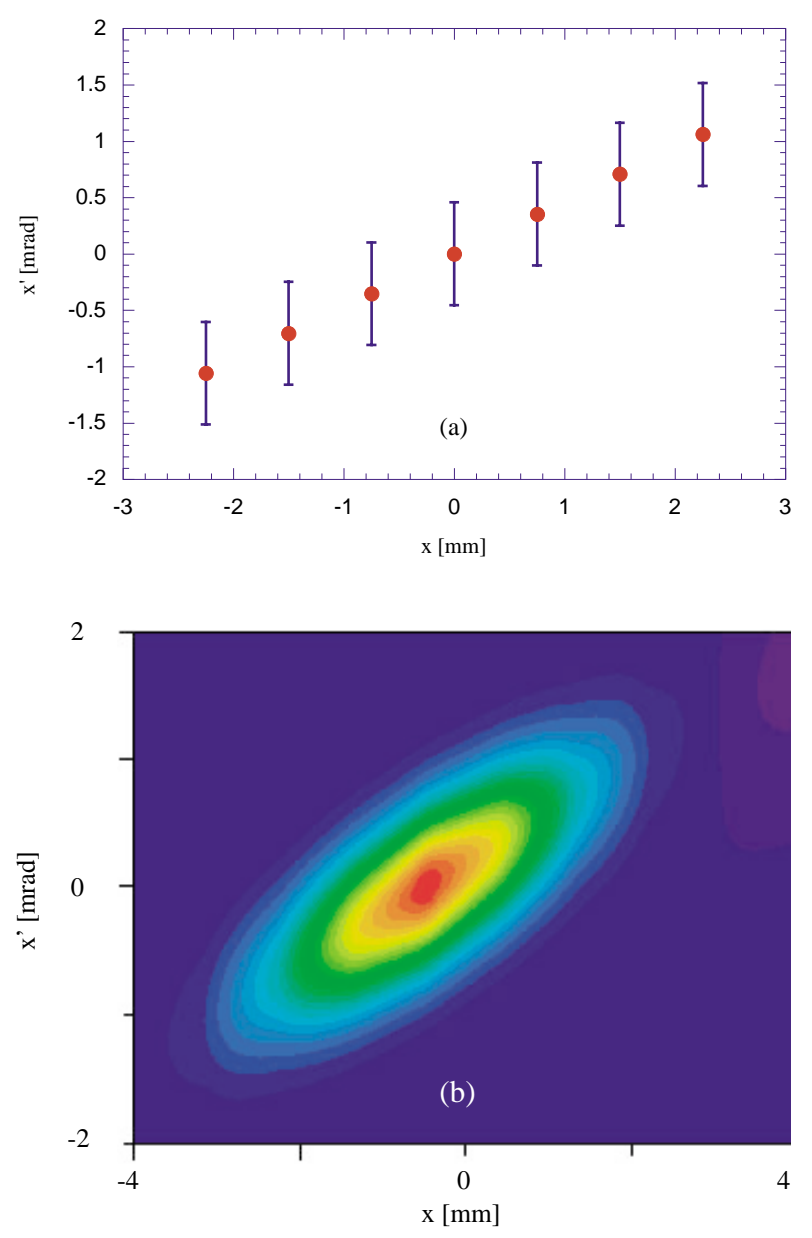

FIG. 2. (Color) (a) Beam trace space constructed from the beamlet intensity profile illustrated in Fig. 1. Each point represents the position of a beamlet in trace space and the error bars indicate the thermal spread of the beamlets. (b) Contour plot representation of the same data. Here the relative weights of the beamlets are resolved. 
and

$$
x_{m, c}^{\prime}=\left.\left(x_{m, c}^{\prime}-x_{c}^{\prime}\right)\right|_{\mathrm{old}}=\left.\left(x_{m, c}^{\prime}\right)\right|_{\mathrm{old}}-\frac{\sum_{m=1}^{N} I_{m} x_{m, c}^{\prime}}{\sum_{m=1}^{N} I_{m}},
$$

where $I_{m}$ is the integrated detected intensity of the $m$ th beamlet.

The second moments of the trace space distribution are obtained from the above determined parameters as

$$
\begin{gathered}
\left\langle x^{2}\right\rangle=\frac{\sum_{m=1}^{N} I_{m} x_{m, c}^{2}}{\sum_{m=1}^{N} I_{m}}, \\
\left\langle x^{\prime 2}\right\rangle=\frac{\sum_{m=1}^{N} I_{m}\left(x_{m, c}^{\prime 2}+\sigma_{m}^{\prime 2}\right)}{\sum_{m=1}^{N} I_{m}},
\end{gathered}
$$

and

$$
\left\langle x x^{\prime}\right\rangle=\frac{\sum_{m=1}^{N} I_{m} x_{m, c} x_{m, c}^{\prime}}{\sum_{m=1}^{N} I_{m}},
$$

where in obtaining the moment $\left\langle x^{\prime 2}\right\rangle$ we have assumed that the beamlet distributions are symmetric about their centroids. From the second moments, we can construct the rms emittance

$$
\varepsilon_{x} \equiv \sqrt{\left\langle x^{2}\right\rangle\left\langle x^{\prime 2}\right\rangle-\left\langle x x^{\prime}\right\rangle^{2}}
$$

as well as the other rms Twiss parameters [11],

$$
\begin{gathered}
\beta_{x} \equiv \frac{\left\langle x^{2}\right\rangle}{\varepsilon_{x}}, \quad \alpha_{x} \equiv-\frac{\left\langle x x^{\prime}\right\rangle}{\varepsilon_{x}}, \\
\gamma_{x} \equiv \frac{\left\langle x^{\prime 2}\right\rangle}{\varepsilon_{x}}=\frac{1+\alpha_{x}^{2}}{\beta_{x}} .
\end{gathered}
$$

The slits serve a secondary purpose which is of central importance in the discussion of beams emitted from rf photocathode guns since these beams are space-charge dominated for almost all relevant energies and beam sizes. The notion of space-charge dominated flow is quantified by comparing the space charge and emittance terms in the rms beam envelope equation for an ultrarelativistic beam $(\gamma \gg 1, \beta=\nu / c \approx 1)$ in a drift space [12]

$$
\sigma_{x}^{\prime \prime}=\frac{\varepsilon_{n}^{2}}{\gamma^{2} \sigma_{x}^{3}}+\frac{I}{\gamma^{3} I_{0}\left(\sigma_{x}+\sigma_{y}\right)},
$$

where $I$ is the peak beam current, $I_{0}=e c / r_{e}$ is the characteristic current, $\varepsilon_{n}=\beta \gamma \varepsilon_{x} \cong \gamma \varepsilon_{x}$ is the normalized rms emittance, and, of course, an analogous equation exists for $\sigma_{y}$. Now, taking the ratio of the second to the first terms on the right-hand side of the envelope equation, and assuming a round beam $\left(\sigma_{x}=\sigma_{y} \equiv \sigma_{0}\right)$, we have a measure of the degree of space-charge dominance over emittance in driving the evolution of the beam envelope,

$$
R_{0}=\frac{I \sigma_{0}^{2}}{2 I_{0} \gamma \varepsilon_{n}^{2}}=2 k_{p}^{2} \beta_{x}^{2} .
$$

In order to illustrate the relationship between the two effects driving the beam envelope, we have rewritten this ratio in terms of the plasma wave number associated with the beam density $n_{b}, k_{p}=\omega_{p} / c \cong \sqrt{4 \pi r_{e} n_{b} / \gamma^{3}}$, and the rms beta function $\beta_{x}$.

As an example, we take parameters typical of the present experiments: a $5 \mathrm{MeV}$ electron beam with current of $100 \mathrm{~A}$, rms beam size of $1 \mathrm{~mm}$, and normalized rms emittance of $4 \mathrm{~mm}$ mrad. This yields a ratio of $R_{0} \cong 75$, and one can see that this beam cannot be emittance dominated until it is focused down to small sizes, $\sigma_{0}<100 \mu \mathrm{m}$. Thus linear transport theory cannot be used to measure the emittance with this type of beam, as we will discuss in detail in our evaluation of the quadrupole scanning technique. Collimation with slits mitigates this situation, however, by creating low current, small $\sigma_{x}$ beamlets that have the same uncorrelated transverse momentum spread as the original beam. Noting that the rms size of a uniform beamlet created by a vertical slit of width $d$ is $\sigma_{x}=d / \sqrt{12}$, and assuming $\sigma_{x} \gg \sigma_{y}$, we have a space-charge dominance ratio for the beamlets,

$$
R_{b}=\sqrt{\frac{2}{3 \pi}} \frac{I}{\gamma I_{0}}\left(\frac{d}{\varepsilon_{n}}\right)^{2}
$$

For the beam parameters of our example, and the choice in our experiments of $d=50 \mu \mathrm{m}$, we see that $R_{b}=$ $0.042 \ll 1$, implying that the beamlets which pass out of the slits are emittance dominated. This process can also be understood in terms of the plasma frequency of the beam and the beta function. Since the beamlets have the same density as the beam before the slits, $k_{p}$ is unchanged by collimation. On the other hand, the beta function of each beamlet is much smaller than that of the beam before collimation, $\beta_{x} \cong \sigma_{x} / \sigma_{x^{\prime}} \Rightarrow(d / \sqrt{12}) / \sigma_{x^{\prime}}$, and for the beamlets the ratio $R_{b}=2 k_{p}^{2} \beta_{x, b}^{2}$ can therefore be made much less than unity.

There are many further technical and physical considerations which must be taken into account in order to arrive at an optimized design for a slit-based phase space measurement system. Since the main point of this article is the overall effect of space charge on the results of emittance measurements, these ancillary concerns are discussed in the Appendix, which is specific to the multislit system implemented in these experiments. Further, more general material on the optimization of this type of instrument is found in the comprehensive review of emittance measurement techniques given in Ref. [8].

\section{QUADRUPOLE SCANNING EMITTANCE MEASUREMENTS}

The quadrupole scanning technique for measuring emittance is well known and widely used in the accelerator physics community [13]. A brief description of the process is given here in order to show its limitations when applied to space-charge dominated beams. One can understand the measurement by considering the evolution of the 
beam's rms Twiss parameters by differentiating Eqs. (9) in a drift length after a thin focusing lens (of focal length $f$ )

$$
\begin{aligned}
& \beta_{x}^{\prime}=2 \frac{\left\langle x x^{\prime}\right\rangle}{\varepsilon_{x}}=-2 \alpha_{x}, \\
& \alpha_{x}^{\prime}=-\frac{\left\langle x^{\prime 2}\right\rangle+\left\langle x x^{\prime \prime}\right\rangle}{\varepsilon_{x}}=-\gamma_{x}, \\
& \gamma_{x}^{\prime}=2 \frac{\left\langle x^{\prime} x^{\prime \prime}\right\rangle}{\varepsilon_{x}}=0,
\end{aligned}
$$

where we used the fact that there are no external forces and ignored the space charge force to set $x^{\prime \prime}=0$. Using Eqs. (13) and applying the thin lens $\left(\alpha_{x 0}\right.$ becomes $\alpha_{x 0}+$ $\left.\beta_{x 0} / f\right)$ gives

$$
\begin{aligned}
\beta_{x}(z)= & \beta_{x 0}-2\left(\alpha_{x 0}+\frac{\beta_{x 0}}{f}\right)\left(z-z_{0}\right) \\
& +\left[\frac{1+\left(\alpha_{x 0}+\frac{\beta_{x 0}}{f}\right)^{2}}{\beta_{x 0}}\right]\left(z-z_{0}\right)^{2} .
\end{aligned}
$$

We notice that the $\beta$ function is quadratic in $z$ as expected. Upon rearranging this equation in terms of $1 / f$ and multiplying it by the emittance, we obtain an equation for the square of the beam size as a function of the focusing strength of the lens,

$$
\begin{aligned}
\sigma_{x}^{2}(z)= & {\left[\sigma_{x 0}^{2}-2 \alpha_{x 0} \varepsilon_{x}\left(z-z_{0}\right)+\gamma_{x 0}\left(z-z_{0}\right)^{2}\right] } \\
& +\frac{2 \sigma_{x 0}^{2}}{f}\left[\frac{\alpha_{x 0}}{\beta_{x 0}}\left(z-z_{0}\right)^{2}-\left(z-z_{0}\right)\right] \\
& +\frac{\sigma_{x 0}^{2}}{f^{2}}\left(z-z_{0}\right)^{2}
\end{aligned}
$$

If we then identify the coefficients of $\left(\frac{1}{f}\right)^{i}$ with the letters $m_{i}$, the emittance can be extracted as

$$
\varepsilon_{x}^{2}\left(z-z_{0}\right)^{4}=m_{0} m_{2}-\frac{m_{1}^{2}}{4} .
$$

With this analysis in mind, the emittance can be obtained by measuring the beam size at a given drift length $\left(L_{d}\right)$ after a quadrupole magnet, scanning through a range of focusing strengths. The same result could have been derived by solving the envelope equation for a drifting beam without space charge:

$$
\sigma_{x}^{\prime \prime}=\frac{\varepsilon_{x}^{2}}{\sigma_{x}^{3}} .
$$

It is important to stress here that the quad scan formalism is based on rms quantities. That is, Eq. (15) holds (without space charge) for arbitrary, evolving beam distributions provided that we are measuring the rms value of the beam size. When considering the effect of space charge in the remainder of this paper, we are looking for gross differences in the rms beam size. This point is seen also in the envelope equations including space charge. In these equations the space-charge term is derived assuming linear self-fields, but extends to other distributions in the rms sense using the concept of equivalent beams [14,15]. This work expands on that of Ref. [9], which points out that the assumption of a Gaussian transverse profile and measurement of full width at half maximum (FWHM) spot sizes is incorrect for nonthermalized, photoinjector beams. For these reasons, all experimental spot sizes quoted in this paper are determined using rms measurements of the distribution, thus avoiding potential problems Gaussian fitting and FWHM-based analyses of the data.

We can now examine how a quadrupole scan behaves in the other extreme, namely the one where we assume the beam has space charge but no emittance. In that case, the envelope equations in $x$ and $y$ become

$$
\begin{aligned}
& \sigma_{x}^{\prime \prime}=\frac{I}{\gamma^{3} I_{0}\left(\sigma_{x}+\sigma_{y}\right)}, \\
& \sigma_{y}^{\prime \prime}=\frac{I}{\gamma^{3} I_{0}\left(\sigma_{y}+\sigma_{x}\right)} .
\end{aligned}
$$

These equations have no exact solution, but we may do an approximate analysis by making the assumption $\sigma_{x}+\sigma_{y} \approx$ const. This approximation is acceptable while $k_{p} L_{d}<1$. In that case we get, through a change of variables $\left(\Sigma=\sigma_{x}+\sigma_{y}, \Delta=\sigma_{x}-\sigma_{y}\right)$,

$$
\begin{aligned}
\Sigma^{\prime \prime} & =\frac{2 I}{\gamma^{3} I_{0} \Sigma} \approx 2 k_{p}^{2} \Sigma_{i} \approx \text { const }, \\
\Delta^{\prime \prime} & \approx 0 .
\end{aligned}
$$

This set of equations is easily solvable and we find

$$
\begin{aligned}
& \Sigma_{f}=\Sigma_{i}+\Sigma_{i}^{\prime} z+k_{p}^{2} \Sigma_{i} z^{2}, \\
& \Delta_{f}=\Delta_{i}^{\prime} z .
\end{aligned}
$$

Using $\sigma_{x}=\frac{1}{2}(\Sigma+\Delta)$, and assuming an initially axisymmetric beam $\left(\sigma_{x, i}=\sigma_{y, i}\right)$, allows a solution of the $x$ envelope equation,

$$
\sigma_{x, f}=\sigma_{x, i}+\left(\sigma_{x, i}^{\prime}-\frac{\sigma_{x, i}}{f}\right) z+k_{p}^{2} z^{2} \sigma_{x, i} .
$$

We see from this that the rms beam size is linear with the focusing strength of the lens, and so the square is quadratic in $1 / f$, just as in the case where space charge is ignored. If one attempts to compute the emittance using the coefficients of powers of $1 / f$, as in the emittance dominated case, the result is zero. This is understandable in the sense that one would expect the beam size to remain linear with $1 / f$ all the way down to zero thickness for a beam with no emittance.

For the measurements described below, neither of these limiting cases is applicable as both the emittance and space charge terms are important in the evolution of the rms beam size. What we have shown here, however, is that one can expect the qualitative outcome of the quadrupole scan to be the same in either regime. In particular, it is notable that, even in the case of space-charge dominated beam dynamics, the algorithm used to extract the emittance from a quadrupole scan [based on Eq. (16)] gives a well-behaved result, and there is no a priori reason that data from these 
scans would be rejected as unphysical. The experimental data shown below demonstrate quad scans that indeed yield good fits to Eq. (16), but which have systematic errors in the resulting calculated emittance. The basis for these errors, which is dependent on the interplay between space charge and emittance forcing of the beam envelope, is discussed below, in the context of the experimental data and modeling.

\section{EXPERIMENTAL SETUP AND PROCEDURE}

The quadrupole scan and multislit-based emittance measurement techniques were compared using the Thomson scattering photoinjector at Lawrence Livermore National Laboratory. The beam line configuration used for these measurements is shown in Fig. 3. The accelerator in this setup was a 1.6 cell, $S$ band, BNL-SLAC-UCLA-LLNL rf photocathode gun [16]. The gun produced a $5 \mathrm{MeV}$ beam whose charge, transverse, and longitudinal spot sizes varied as given in Table I and described below. An emittance compensating solenoid after the gun was used to control the rf defocusing at the gun exit and allowed us to select a reasonable beam size at the emittance slits and quadrupole. The magnetic field at the cathode was nulled with an identical bucking solenoid placed upstream of the cathode. The charge was measured using an integrating current transformer. The laser injection phase was monitored during the experiments by mixing the low level rf derived from the laser oscillator with that from a probe in the gun full cell. Long time scale drifts in the rf phase were corrected for all measurements with a manual phase shifter.

A Ti:sapphire-based laser system was used to produce the UV pulses necessary for photoelectron emission in the gun. As the injector is intended for integration with an rf linear accelerator (linac) and a multi-Joule short-pulse laser system $[17,18]$, the beam produced by the photoinjector must be synchronized with the high power laser pulses. The photoinjector laser system (PLS) is seeded by stretched pulses from the Falcon laser system so that the linac can be synchronized to the Falcon master oscillator. The seed light is introduced into the regenerative amplifier
TABLE 1. Electron beam parameter range used for emittance measurements.

\begin{tabular}{lc}
\hline \hline \multicolumn{1}{c}{ Parameter } & Range \\
\hline Electron beam charge & $50-300 \mathrm{pC}$ \\
rms laser spot size & $0.5-2 \mathrm{~mm}$ \\
rms laser pulse length & $2.5-6 \mathrm{psec}$ \\
\hline \hline
\end{tabular}

(regen) of the photoinjector system via single-mode, polarization-preserving fiber that runs from the Falcon stretcher output to the basement laboratory containing the PLS. The timing for the Falcon, linac, and PLS derive from the Falcon master oscillator. In addition to the light pulse, timing signals from the Falcon timing rack are sent to the PLS to trigger the yttrium-aluminum-garnet (YAG) pump laser and regenerative amplifier $Q$ switch. The PLS consists of a fiber-seeded regenerative amplifier, a multipass power amplifier, a pulse compressor, and frequency conversion crystals for frequency tripling the $800 \mathrm{~nm}$ laser pulse. The two laser amplifiers, regen and multipass, are pumped by a single, frequency-doubled, $Q$ switched YAG laser that puts out $300 \mathrm{~mJ}$ of $532 \mathrm{~nm}$ light in an $8 \mathrm{nsec}$ pulse. The output beam of the multipass amplifier is expanded and sent to the grating compressor.

We measured the pulse width at the output of the grating compressor using a single-shot autocorrelator. The desired pulse length of the UV pulse is a few psec, which can be obtained by varying the distance between the gratings in the compressor. We attempted to directly measure pulse width vs grating separation but found that the autocorrelator gives good results for only pulses $\sim 1 \mathrm{psec}$ or less. Consequently, we estimate the pulse width for $\Delta T>1 \mathrm{psec}$ using various techniques. For pulse lengths $<1$ psec the autocorrelator appears to give accurate results. The shortest pulse we measured was $150 \mathrm{fsec}$, which corresponds to a time-bandwidth-limited pulse.

By varying the difference in grating separation from the minimum pulse length position $\Delta b$ between the gratings in the pulse compressor we can continuously adjust the pulse length in the IR from 184 psec to 150 fsec. This variation

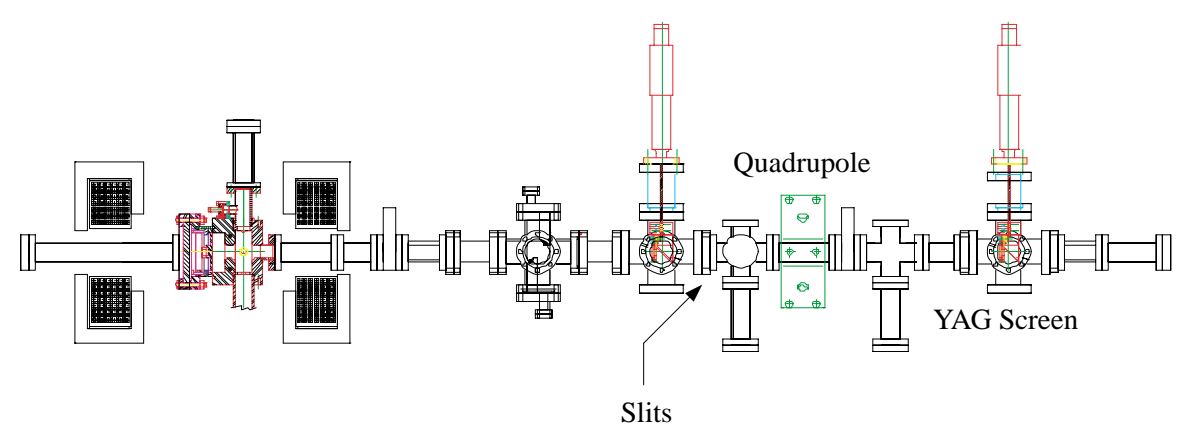

FIG. 3. (Color) Schematic of the LLNL Thomson scattering beam line used in the emittance measurements. 
was used to change the beam intensity as it was launched at the photocathode, thus also varying the plasma frequency of the beam. Three separate methods of modeling the compressor all give the same result, which is that the grating separation tuning $\Delta T / \Delta b \sim 0.2 \mathrm{psec} / \mathrm{mm}$, at $1 \omega$. The $3 \omega$ intensity is proportional to the $1 \omega$ intensity cubed, and, as a result, a Gaussian-shaped pulse narrows up in time, i.e.,

$$
\Delta T_{3 \omega}=\frac{\Delta T_{1 \omega}}{\sqrt{3}} .
$$

Based on this calculation, we expect the pulse width of the $3 \omega$ light to be given by

$$
\Delta T_{3 \omega}=(0.11 \mathrm{psec} / \mathrm{mm}) \times \Delta b(\mathrm{~mm}) .
$$

For the multislit-based emittance measurements we chose, based on the arguments given above and in the Appendix, a set of stainless steel collimating slits with a $50 \mu \mathrm{m}$ slit width, $0.75 \mathrm{~mm}$ separation, and $5 \mathrm{~mm}$ depth. The drift length from the slits to the measurement screen was approximately $50 \mathrm{~cm}$. For a beam size of $1-2 \mathrm{~mm}$ on the slits and at $5 \mathrm{MeV}$, this allowed us to measure a maximum normalized emittance of $12 \mathrm{~mm}$ mrad using the slits. The slits were mounted on an insertable, rotatable actuator powered by stepper motors. This allowed us to align the slits to the beam, insuring the proper angle for maximum acceptance of the slits. A $0.5 \mathrm{~mm}$ thick YAG:Ce crystal was used as the intercepting screen for both types of measurements. The beam images produced at the crystal were captured by a video camera and digitized by a computer controlled frame grabber. Once captured, the beam images were analyzed on-line in the case of the multislit measurements, and saved for latter analysis in the case of the quadrupole scan.

Given the apparatus described above, the experimental procedure was to set the compressor gratings to a given separation distance and measure the dimensions of the laser and electron beams as well as the charge and injection phase. This was important because it allowed us to calculate the plasma frequency of the beam in order to compare our measurements with simulation and analytical models. Once these parameters were known, the beam emittance was measured using both the multislit and the quad scanning techniques. Table I lists the range in beam parameters over which the emittance measurements were done.

\section{EXPERIMENTAL RESULTS}

The quad scan and multislit measurements were performed for seven different electron beam pulse lengths. Figure 4 shows one image of a beam horizontally focused by the quadrupole. The horizontal $\mathrm{rms}$ size of the beam is $140 \mu \mathrm{m}$. The figure also shows a representative result of one of the quadrupole scans. Figure 5 shows the
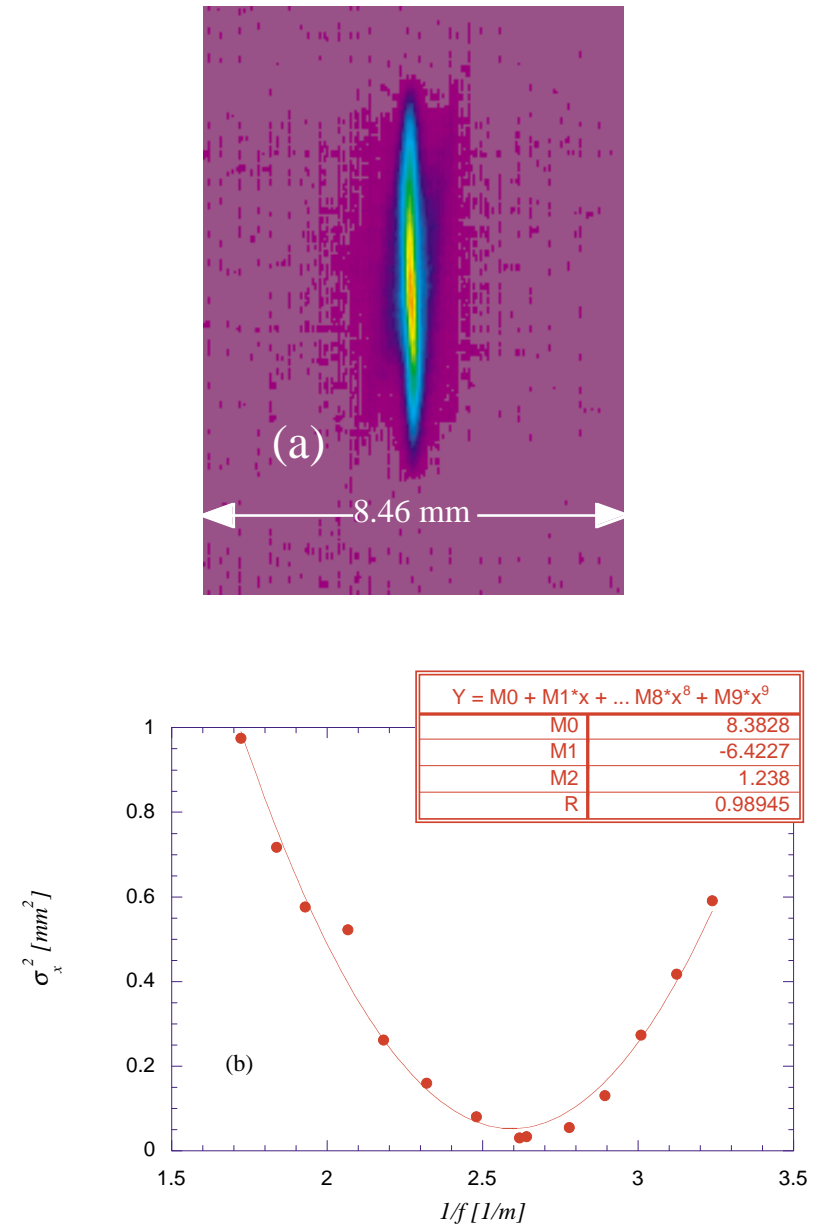

FIG. 4. (Color) (a) False color image of a beam image used in a quadrupole scan. (b) Result of one quad scan. The normalized horizontal emittance found from the curve fit is $9.6 \pm 1.1 \mathrm{~mm} \mathrm{mrad}$.

data found using the slits for the same grating separation as used in Fig. 4. The emittance calculated via the quad scan measurement is higher than with the emittance slits. This pattern was repeated in all of the measurements we performed.

As we argued above, we expect the result of a quadrupole scan to depend not only on the emittance but also the strength of the space-charge defocusing forces encountered by the beam through the drift region. For this reason we plot in Fig. 6 the results of the measurements as a function of the plasma wave number of the beam at the quadrupole multiplied by the drift length between the quadrupole and the screen. The plasma wave number is determined through simulation, using the measured properties of the laser and the total charge launched, as discussed further in the following section [5]. For this range of beam parameters, the quad scan gives consistently higher values for the emittance than the multislit measurement. 

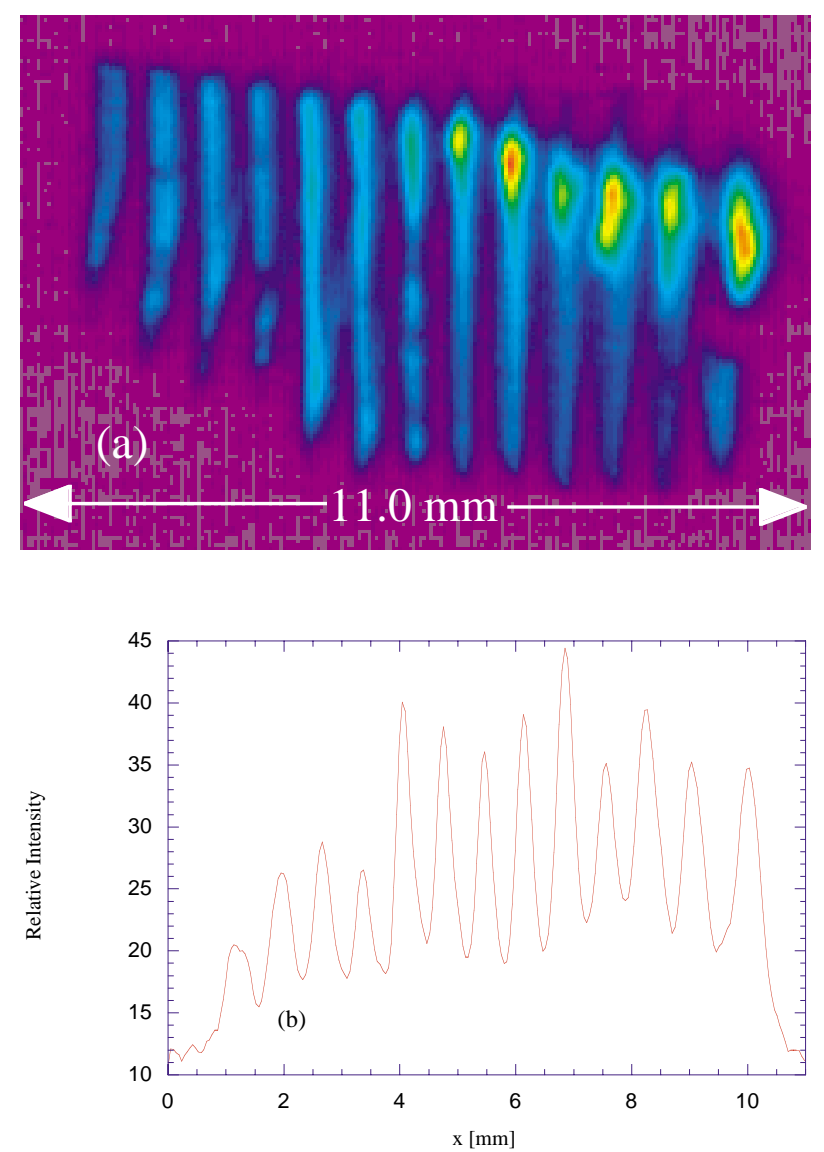

FIG. 5. (Color) (a) False color image of the beam passing through the collimating slits. (b) Intensity graph found by summing the vertical pixel values at a given horizontal position. The normalized horizontal emittance calculated from this plot is $6.9 \pm 0.7 \mathrm{~mm}$ mrad.

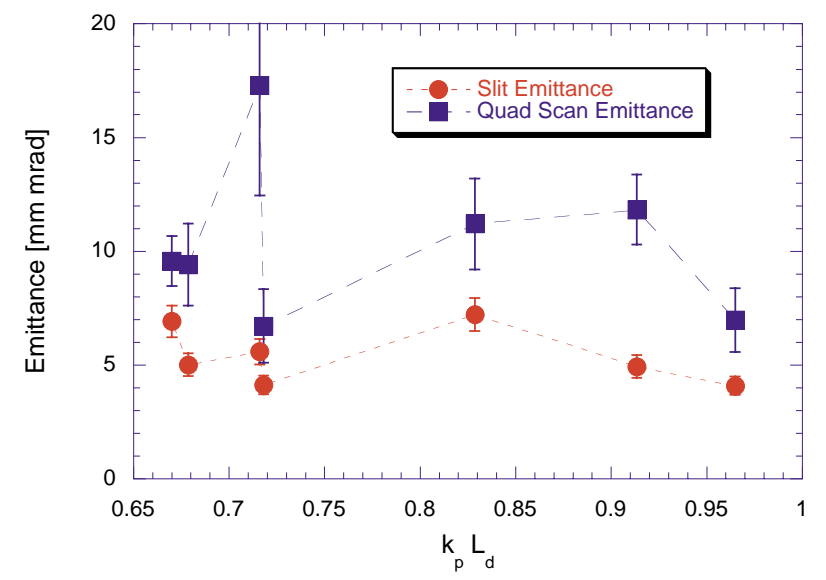

FIG. 6. (Color) Plot comparing the different emittance measurement techniques for varying beam intensities. The strength of the space-charge forces is parameterized in the scan by the product of the drift length between the quadrupole and detector and the plasma wave number at the quad.

\section{ANALYSIS}

The recording of laser spot size, length, and energy for each set of emittance measurements allowed us to accurately simulate the acceleration and focusing of the beam through the 1.625 cell gun. These simulations were done using PARMELA [19], a multiparticle code which includes collective (space-charge) forces. In these simulations, a beam derived from injection of a Gaussian longitudinal and transverse laser pulse shape was used. The purpose of these simulations was both to get a base line expectation value for the emittance of the beam and to find the spatial dimensions of the beam at the position of the quadrupole. The results of the PARMELA simulations are shown in Fig. 7. We see from the figure that the emittance predicted by PARMELA is generally slightly lower than that of the multislit measurements, but follows the same trend from measurement to measurement. This indicates that the small variation in the multislit emittance values at different beam densities is due, at least in part, to changes in the emittance compensation process caused by different beam densities at the cathode, as could be expected.

At this point we simulated the quadrupole scan procedure with space charge included in the simulation. This was done in three different ways. The first was to use the PARMELA beam distributions from the previous simulations at the position of the quadrupole and iteratively simulate their propagation in the drift region between the quad and the measurement screen for different quad strengths. In these PARMELA runs, we changed the space-charge routine to a point-to-point Coulomb method, which is much less accurate than the standard radial mesh algorithm, but was needed to model the beam when it becomes highly asymmetric after quad focusing. In order to avoid the noise inherent in the point-to-point calculations, we also employed HOMDYN, which is an envelope code that has been successfully benchmarked against multiparticle codes [20]. Finally, we also simulated the quad scans by numerically

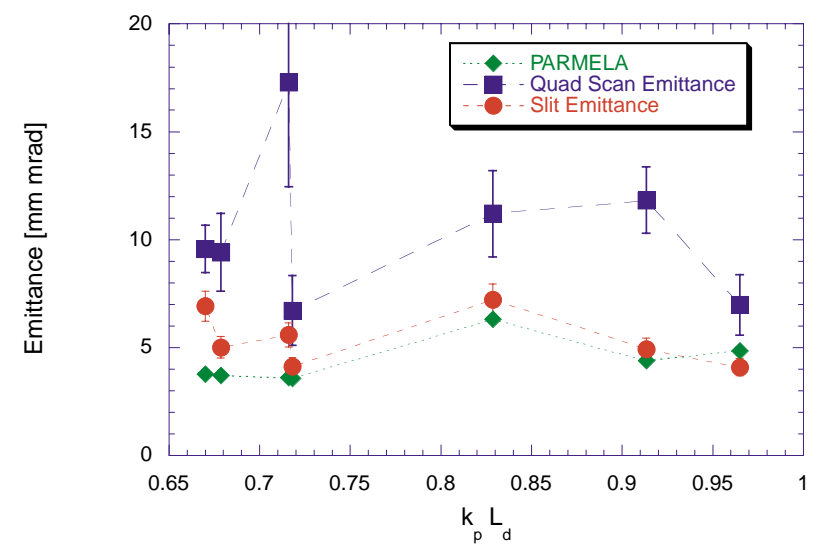

FIG. 7. (Color) PARMELA simulations of the emittance at the quadrupole and the measured emittance using both measurement methods. 
integrating the 2D rms envelope equations for the beam including space charge,

$$
\begin{aligned}
& \sigma_{x}^{\prime \prime}=\frac{\varepsilon_{n}^{2}}{\gamma^{2} \sigma_{x}^{3}}+\frac{I}{\gamma^{3} I_{0}\left(\sigma_{x}+\sigma_{y}\right)}, \\
& \sigma_{y}^{\prime \prime}=\frac{\varepsilon_{n}^{2}}{\gamma^{2} \sigma_{y}^{3}}+\frac{I}{\gamma^{3} I_{0}\left(\sigma_{x}+\sigma_{y}\right)} .
\end{aligned}
$$

In the case of both the HOMDYN and envelope equation simulations, the emittances $\varepsilon_{n}$ [cf. Eq. (24)] were taken to be that measured by the slits, the initial beam sizes were taken from PARMELA, and the beam currents taken from measurement data. In all three cases the simulations gave similar results, and the less noisy (but still three dimensional, and sensitive to differential slice dynamics [4,5]) HOMDYN results are shown in Fig. 8. In order to avoid confusion about the meaning of the emittance term in Eq. (24), we specify at this point that the symbol $\varepsilon_{n}$ will indicate the normalized thermal emittance. The thermal component to a full measured emittance is due to uncorrelated motion, as opposed to the apparent emittance observed from residual correlations between longitudinal and transverse phase space in poorly compensated beams. Thus we are assuming in the integration of Eq. (23) that the measured multislit emittance is roughly equal to the so-called slice emittance. This point is physically meaningful, as an emittance which was entirely based on longitudinal-transverse phase space correlations, with vanishing slice emittance, would not give rise to effects we have observed.

As we see from Fig. 8, the emittances derived from the simulated quad scans are indeed higher than the actual emittances used in the simulations, as expected. In addition, they agree reasonably well with the results of the quad scan measurements.

The parameter $k_{p} L_{d}$ was chosen to plot against the emittance results because it describes the degree to which the plasma nature of the beam influences its motion in the experiments. In cases where the beam evolves in a purely space-charge dominated way (such as the emittance com-

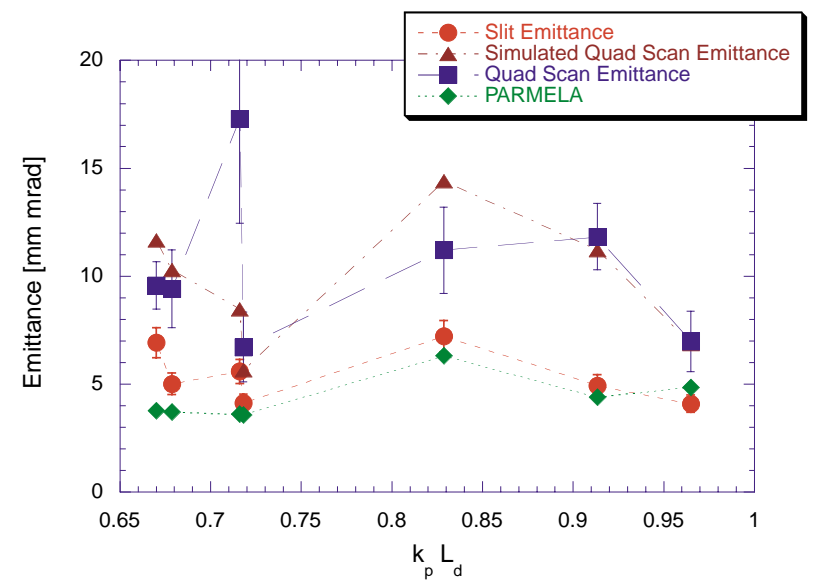

FIG. 8. (Color) Simulation of the quadrupole scan plotted with the PARMELA simulations and the measured emittance data. pensation process), $k_{p} L_{d} / 2 \pi$ would be the number of plasma oscillations that the beam undergoes. However, in the case of the quad scan, the beam's size at the end of the drift is also affected by emittance, especially at points where the beam has gone through a tight waist. In that case, the relative strength of the emittance and spacecharge terms $\left(R_{0}\right)$ in the envelope equation are of interest. The ratio of these forcing terms can be characterized by the beta function times the plasma wave number, and Eq. (11) shows that $k_{p} \beta=\sqrt{\frac{R_{0}}{2}}$. In Fig. 9 we plot the path of the experimental data in the plane defined by $k_{p} \beta$ and $k_{p} L_{d}$. The background of the plane is a contour plot of simulated quad scan emittances using the envelope integration model, with a constant thermal emittance for each point in the plane. We see from the figure that some data points that happen to be close together in $k_{p} L_{d}$ have significantly different values of $k_{p} \beta$. This effect explains in part the drastic difference in emittance between close data points in Fig. 8. Note also that the lower right-hand corner of the graph has values of emittance that are lower than the value that was used to integrate the envelope equations. This point will be discussed further below.

It is instructive at this point to look again at the quad scan procedure to see why the data shows and simulations predict erroneous numbers for the emittance. In particular, we can see both in the data and in simulations that there is an asymmetry in the curves about their minima. In Fig. 10 the quad scan data from Fig. 4 is replotted, and the data points on either side of the minimum are fitted to different curves. We see from the figure that the points before the minimum spot size follow a path with less curvature than the points occurring after the focus. The second part of the figure shows this effect more dramatically. In that case the plot points come from a simulation of a quad scan assuming conditions similar to the experiment, but with a higher beam current of several hundred amps.

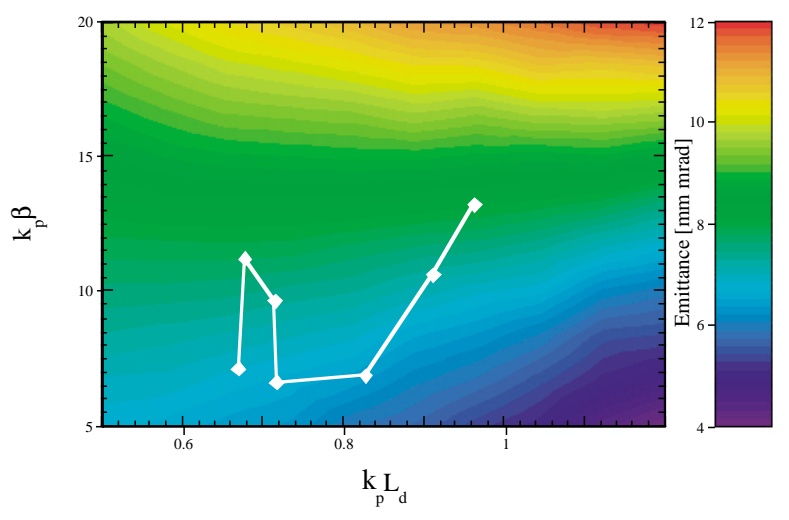

FIG. 9. (Color) Contour plot of the simulated quad scan emittance over a range of both $k_{p} L_{d}$ and $k_{p} \beta$. The white plot points locate the positions of the experimental data. The normalized emittance used as input to the simulations was $5 \mathrm{~mm} \mathrm{mrad}$, while the output emittance values range from 4 (deep blue) to $12 \mathrm{~mm}$ mrad (red). 

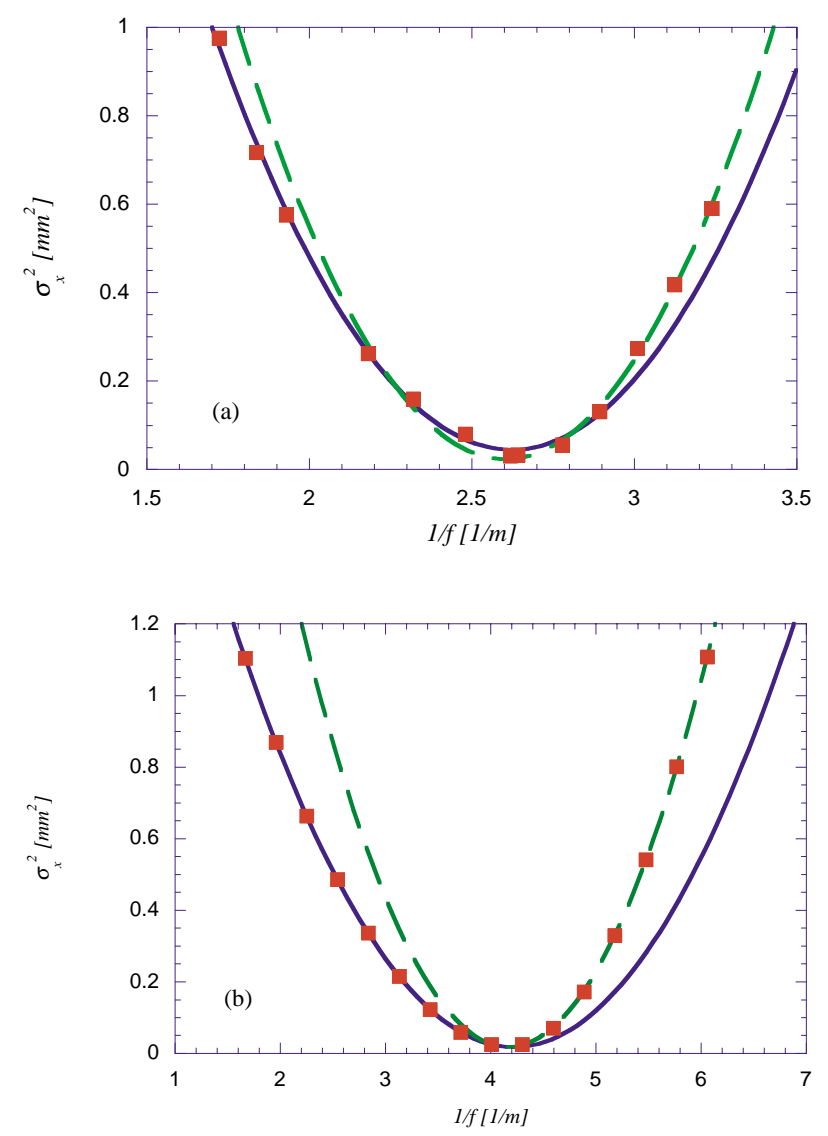

FIG. 10. (Color) (a) Quad scan data as shown in Fig. 4(b). Here we apply different fits to the data before and after the focus. (b) Simulation of a quad scan with an extremely space-charge dominated beam. Again, we show the two fits on either side of the beam waist to illustrate the asymmetry in the simulation points.

This asymmetry in the data about the minimum spot size is a manifestation of the fact that the evolution of the beam through the drift is very different for data points on opposite sides of the minimum. For points on the right-hand side of the curve (weaker focusing) the beam size is deflected appreciably only by space charge. For points in the curve at and to the left of the waist (stronger focusing) there occurred at some position a thermal emittance dominated beam waist. In the region of that waist, the emittance force "turns on" and applies an extra kick to the beam size that deflects it away from the path it would take due only to space charge. The stronger the quadrupole focuses (larger $1 / f)$, the stronger this thermal emittance kick will be and the further upstream of the measurement screen it occurs. Figure 11 illustrates this behavior, by showing the results of a simulation with and without the emittance term included in the envelope equations. We see here that for points on the left-hand side of the minimum, the simulations with and without emittance agree very well, indicating that the integrated motion of the beam is indeed purely dominated by space charge. However, the points at and after the minimum diverge rapidly from the path that the

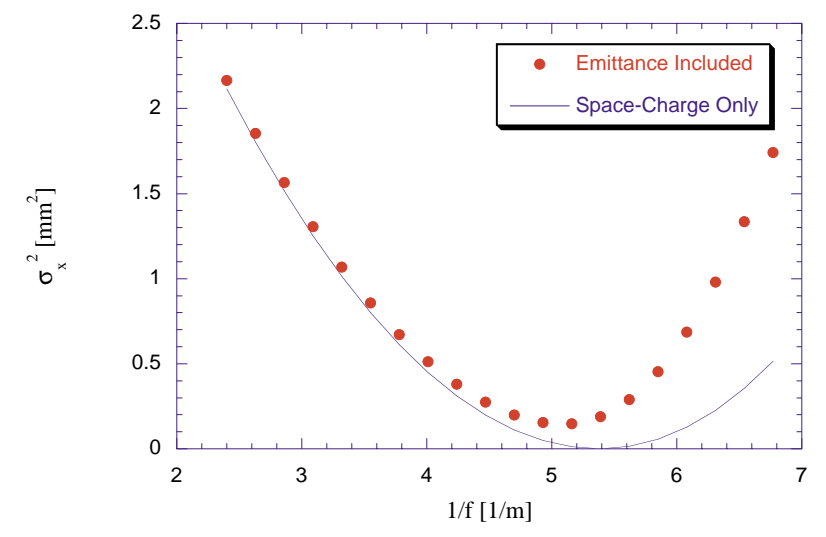

FIG. 11. (Color) Simulations of a quad scan with and without emittance. The solid line represents a simulation without the emittance forces, while the data points show a simulation of the full envelope equations.

simulation with space charge alone predicts even though the beam is space-charge dominated over most, but not all, of its trajectory.

It is interesting to note that both a purely thermal emittance dominated and a purely space-charge dominated scan would produce a $\left(\sigma_{x}^{2}, 1 / f\right)$ curve which is symmetric about the minimum $\sigma_{x}$. The asymmetry observed in the mostly space-charge dominated quadrupole scan is a result of both space-charge and thermal emittance effects asserting themselves in the measurement.

Given this problem, in which points on one side of the curve are space-charge dominated, while on the other side they are affected by emittance, it is certainly no surprise that the emittance computed from these curves is not correct. In addition, the asymmetry of the curve introduces a problem in the consistency of the result. That is, since the computation of the emittance requires us to fit a parabola to a curve that is of higher order, the fit parameters will depend on the portion of the curve used for the fit. To illustrate this point, consider a single quad scan simulation as shown in Fig. 12(a). The range of focal lengths we use for the fit is in principle arbitrary; however, it is reasonable to impose the conditions that the simulation points are equally spaced in $1 / f$ and that the end points give the same beam size, which is about $1 \mathrm{~mm}$. These are the conditions we used for simulations in this paper. We can see how the computed emittance varies when we change the start and stop point of the fit. Figure 12(b) shows the emittances calculated from the simulation data of Fig. 12(a) with the horizontal and vertical axes representing the starting and stopping points of the fit, respectively.

There are two noteworthy features in Fig. 12(b). The first is that it shows both regions above and below the input value of the emittance. In fact, the violet regions in the upper half of the plot represent points where the computed emittance is imaginary [this happens if the right-hand side as determined from the scan parameters of Eq. (16) is negative]. The other point is that the upper left corner of 

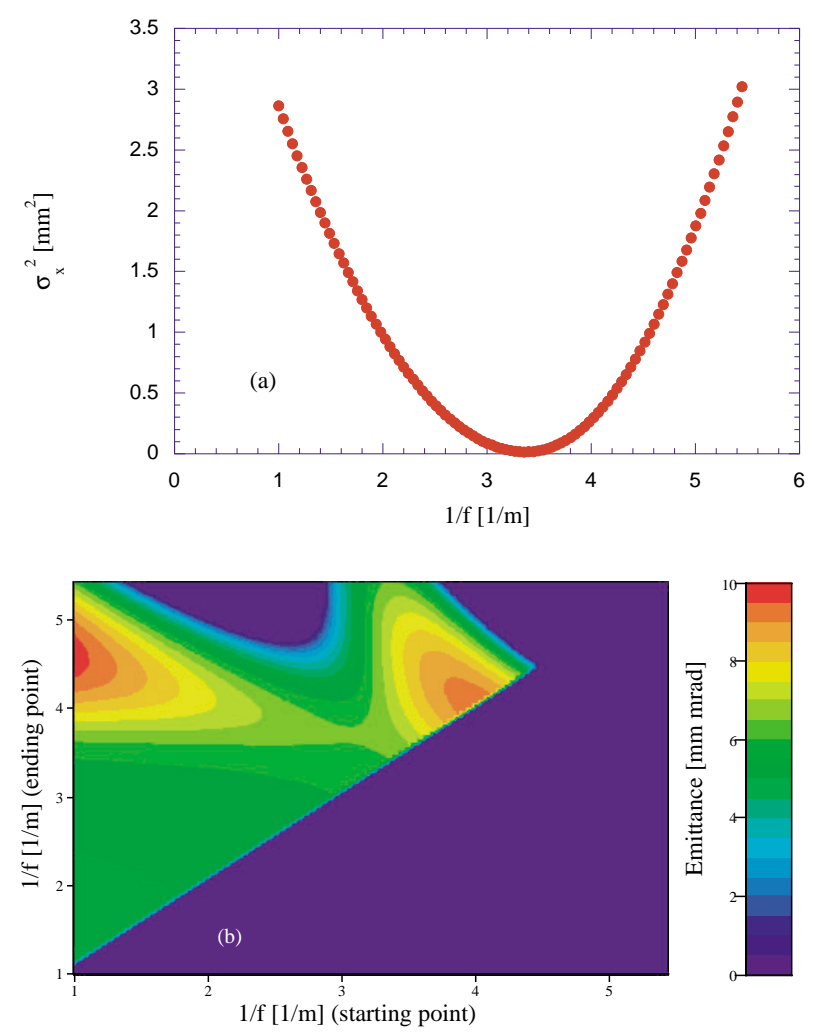

FIG. 12. (Color) (a) Quad scan simulation used to perform the fitting in (b). The emittance used in the simulation is $4 \mathrm{~mm} \mathrm{mrad}$. (b) Contour plot of the emittance calculated by fitting the data in (a). The portion of the graph below the diagonal is unused (this half would be the mirror image of the upper half.) Here, red represents an emittance of $10 \mathrm{~mm}$ mrad and violet is imaginary (the square of the derived emittance is negative).

the graph is the region where the gradient in emittance values is the steepest. This is also the region that we initially assumed to be the most reasonable. The lower left-hand corner of the plot seems to be a stable region, but it is not an accurate solution. Points in this corner fit only the first part of the curve, and that part gives only information about space charge, as shown in Fig. 11. This strict dependence of the computed emittance on the range of fit points is another factor that may help to explain the inconsistency in the quad scan measurements. This effect was not considered at the time the measurements were performed, and for the majority of them slightly more data points were taken before the minimum of the curve than after.

\section{CONCLUSIONS}

Two different emittance measurement techniques were discussed in this paper: the multislit-based measurement and the quadrupole scan. For the highly space-charge dominated beams of interest here, we reviewed how the slit collimation of the beam into beamlets reduces the quantity $k_{p} \beta$ sufficiently to allow the beamlets to expand under the influence of emittance and not space charge. In the ex- periment preformed at the Thomson source photoinjector at LLNL, we found that the emittances measured with the multislit system were reasonably independent of the beam intensity, and agreed well with the PARMELA simulations.

In contrast, we found that the quadrupole scanning procedure was ill-suited to measuring the emittance of these beams. In addition to the issues associated with multishot measurements in systems with notable shot-to-shot charge fluctuations, the fundamental problem with the quadrupole scan is that the beam evolves under the influence of both space-charge and emittance effects. In the Livermore experiments we found that the emittance measured with the quad scan was consistently higher than the multislit measurements and PARMELA predictions. In addition, simulations of the quad scan for the beam parameters of the measurements also show higher values for the emittance, and reproduce the systematic dependence of the measured emittance on the quantity $k_{p} L_{d}$.

Simulations show that when $k_{p} L_{d}$ is of the order of unity or greater, space-charge forces are large enough to significantly alter the evolution of the beam in the drift region of the scan. Further, when the quantity $k_{p} \beta$ is larger than unity (indicating the degree to which the spacecharge forces are dominant over the emittance effects), the quadrupole measurement of the emittance will have notable errors. For $k_{p} \beta \gg 1$, the emittance measurement is no longer valid at all, but is really only a measure of the intricate interplay between the space-charge and emittance effects during the focused trajectory. Thus the conditions $k_{p} L_{d}>1$ and $k_{p} \beta>1$ are theoretical tools for determining the range of parameters over which the validity of the quadrupole scan is no longer guaranteed. Perhaps an even more useful method that can test for possible problems in a quadrupole scan is the examination of the scan data itself. When the $\left(\sigma_{x}^{2}, 1 / f\right)$ curve is no longer symmetric in $1 / f$ about the minimum in $\sigma_{x}^{2}$, this is a clear signature that the combined space-charge/emittance dominated beam evolution produces an unreliable emittance measurement. It is both inaccurate and imprecise, as the exact value of the emittance derived from the $\left(\sigma_{x}^{2}, 1 / f\right)$ curve depends on the number of points on either side of the minimum that are used in the fit.

It may be noted that, as experimental progress is made in lowering the beam emittance obtained from rf photoinjectors, the problems noted here with quadrupole scans will be exacerbated. In recognition of such difficulties, most laboratories use slit-based measurements for low energy $(<10 \mathrm{MeV})$ emittance diagnosis. The present results imply that quadrupole scans may be problematic at energies much higher than $10 \mathrm{MeV}$.

\section{ACKNOWLEDGMENT}

This work was performed under the auspices of the U.S. Department of Energy by the Lawrence Livermore National Laboratory under Contract No. W-7405-Eng-48. 


\section{APPENDIX}

We survey in this Appendix a series of design considerations which impact the choice of slit parameters one deploys in the phase space measurement system, and which dictate the resolution of the device. Many of the issues discussed here are also examined in Ref. [8], but since Lejeune and Aubert do not analyze the slit array in particular, a review of the technical considerations one needs to implement the slit-based system is given here. We begin by examining those parameters that have to do with the angular acceptance of the slits. The depth of the material used to intercept the beam is dictated by our desire to either stop the beam or scatter it sufficiently so that it does not affect the measurement of the nonintercepted beamlets. The stopping distance of the beam is approximately

$$
L_{s}=\frac{E}{\frac{d E}{d x}} \approx \frac{E(\mathrm{MeV})}{1.5\left(\mathrm{MeV} \mathrm{cm}^{2} \mathrm{~g}^{-1}\right) \rho\left(\mathrm{g} \mathrm{cm}^{-3}\right)}
$$

for an initially minimum ionizing particle. It is straightforward to stop a $5 \mathrm{MeV}$ beam, such as is encountered in this paper, but with an energy much above $10 \mathrm{MeV}$, the length of the slits for total stopping may become impractical. In order to deal with this we rely on multiple scattering effects in the slit material. The beam scatters off of nuclei as it slows down from ionization losses, and the final rms angle associated with the beam after propagating a distance $L$ in the stopping material is approximately

$$
\begin{aligned}
\theta_{\mathrm{sc}} & \cong \frac{21}{E(\mathrm{MeV})} \sqrt{\frac{L_{s}}{L_{r}}\left(\frac{1}{L_{s}-L}-1\right)} \\
& \cong \frac{21}{E(\mathrm{MeV})} \sqrt{\frac{L}{L_{r}}},
\end{aligned}
$$

where $L_{r}$ is the radiation length in the material, and we assumed the stopping distance is much larger than the material thickness. In order to reduce the background signal from scattered (but not stopped) electrons, it is useful to conservatively require a multiple scattering angle in excess of 0.5 , or approximately,

$$
L_{s} \cong L_{r}\left(\frac{E(\mathrm{MeV})}{42}\right)^{2} .
$$

Once the slit depth $L$ has been chosen, one can examine the angular acceptance of the slits. The first step is to specify an rms beam angle associated with the finite beam emittance which, assuming we place the slits at a waist, is

$$
\phi=\frac{\varepsilon_{n}}{\gamma \sigma_{0}} .
$$

The assumption of a beam waist is invariably a good approximation, as one must be careful not to introduce a large correlated beam angle in order to make sure that all offsets $x$ in the beam pass the slit aperture well. Thus we are left to consider the uncorrelated angles given by Eq. (A4), which must be chosen to be less than one-fourth the angular aperture of the slits, $\phi<d / 4 L$, and preferably even smaller.

The slit separation $w$ is chosen to be much larger than the slit width $d$ and smaller than the beam size to ensure that we can resolve the beam. In our case, the slit width is taken to be $0.75 \mathrm{~mm}$. This width must also be consistent with not allowing the beamlets to overlap at the detection plane, a condition that depends on the distance of the drift to the screen $L_{d}$. The ratio to the beamlet widths to their separation, which should be smaller than unity, is

$$
R_{w s}=2 \frac{L_{d} \phi}{w}
$$

while the ratio of the beamlet rms size at the phosphor to its size at the slit, which should be larger than 1 to achieve resolution of the uncorrelated angular spread in the beam, is

$$
R_{s p}=\sqrt{12} \frac{L_{d} \phi}{d} .
$$

Since one of these ratios should be small and the other large compared to unity, if we set their geometric average equal to unity $\left(R_{s p} R_{w s}=1\right)$, we can optimize the drift length to be

$$
L_{d} \cong \frac{\sqrt{d w}}{3^{1 / 4} 2 \phi},
$$

which for the example parameters given above yields $L_{d} \approx$ $50 \mathrm{~cm}$. This optimum is of course quite broad, so one is free to choose a more convenient value if one desires to measure larger or smaller emittances, as discussed below.

Once the drift length is specified, there is another criterion which should be examined for the diagnostic to give unambiguous results: the contribution to the measured emittance from the residual space-charge forces between beamlets is smaller than that due to the true uncorrelated angular distribution at the slits. Again assuming the slits are at a waist (this gives the highest estimate of the space-charge effect), we have

$$
R_{b}^{\prime}=\frac{2 I}{\gamma^{2} I_{0}} \frac{d L_{d}}{w \varepsilon_{n}} .
$$

Again, this quantity must be much smaller than 1 . For our present design it is about 0.25 , but it should be noted that for Hartman's measurements it was in fact greater than 1 .

The subject of slit scattering is a bit complicated, but a detailed calculation using EGS [21] is not necessary if estimates that the signal-to-noise due to slit scatter is not of the order of 100 or less. Theoretical guidelines in this calculation have been developed by Courant [22] and Burge and Smith [23]. Modifying Courant's criterion for energy discrimination, we pick the effective depth of the maximum of the relevant slit scattered flux to occur when the multiple scattering angle is equal to the acceptance half-angle of the slits, 


$$
l_{\mathrm{eff}}=L_{r}\left(\frac{21 d}{E(\mathrm{MeV}) 2 l}\right)^{2}
$$

and the increase in effective slit width is given by

$$
d_{\mathrm{eff}}=\frac{2}{\sqrt{3 \pi}} \frac{l_{\mathrm{eff}}^{3 / 2}}{w_{c}},
$$

where

$$
w_{c}^{2}=\frac{A}{Z^{2} \pi N_{A} \rho}\left(\frac{E}{2 e^{2}}\right)^{2} \ln \left(181 Z^{1 / 3}\right)^{-1},
$$

and $N_{A}$ is Avogadro's number. The minimum signal-tonoise for the detected beam intensity at the phosphor is therefore

$$
\frac{S}{N} \geq \frac{\sqrt{3 \pi} d w_{c}}{2 d_{\mathrm{eff}} l_{\mathrm{eff}}^{3 / 2}} .
$$

For most cases of interest, Eq. (A12) gives a value much in excess of unity. It should be noted, however, that a misalignment of the slits can generate anomalously large slit scattering effects, and thus care must be taken to avoid this situation.

Once the general layout of the slit system is specified by the above-listed optimization, one may examine the resolvable emittances in the measurement device. The maximum emittance one may measure is obtained in the situation where the angular aperture of the slits is entirely filled (assuming that the requirement of the ratio $R_{s p}<1$ is satisfied), so that

$$
\varepsilon_{n, \max }=\frac{\gamma \sigma_{0} w}{\sqrt{12} L_{s}}
$$

Note that this maximum emittance is proportional to the beam size, and thus the total number of slits in the mask $N_{s}$. On the other hand, the minimum emittance which can be resolved is given by

$$
\varepsilon_{n, \min }=\frac{\gamma \sigma_{0} d}{\sqrt{12} L_{s}}
$$

As a practical matter, it should be noted that great care needs to be taken in the machining specifications for the slits, to ensure that they are flat over the entire surface parallel to the beam propagation. This is accomplished in the present experiments by electron discharge machining. The slits are mounted on a precision insertable-rotatable (2000 steps per rotation) actuator which is driven by a stepping motor. This level of precision is needed to eliminate unwanted angles between the beam centroid propagation and the slit normal directions.

[1] J. S. Fraser and R. L. Sheffield, IEEE J. Quantum Electron. 23, 1489 (1987).

[2] K. J. Kim, Nucl. Instrum. Methods Phys. Res., Sect. A 275, 201 (1989).

[3] B. E. Carlsten, Nucl. Instrum. Methods Phys. Res., Sect. A 285, 313 (1989).

[4] Luca Serafini and J. B. Rosenzweig, Phys. Rev. E 55, 7565 (1997).

[5] S. G. Anderson and J. B. Rosenzweig, Phys. Rev. ST Accel. Beams 3, 094201 (2000).

[6] X. Qiu et al., Phys. Rev. Lett. 76, 3723 (1996).

[7] J. B. Rosenzweig et al., Nucl. Instrum. Methods Phys. Res., Sect. A 410, 437 (1998).

[8] Claude Lejeune and Jean Aubert, Adv. Electron. Electron Phys., Suppl. 13A, 159 (1980).

[9] B.E. Carlsten et al., Nucl. Instrum. Methods Phys. Res., Sect. A 331, 791 (1993).

[10] S. Hartman et al., Nucl. Instrum. Methods Phys. Res., Sect. A 340, 219 (1994); J. Rosenzweig et al., Nucl. Instrum. Methods Phys. Res., Sect. A 341, 379 (1994).

[11] R. Q. Twiss and N.H. Frank, Rev. Sci. Instrum. 20, 1 (1949).

[12] Martin Reiser, Theory and Design of Charged Particle Beams (Wiley, New York, 1994).

[13] Helmut Wiedemann, Particle Accelerator Physics: Basic Principles and Linear Beam Dynamics (Springer-Verlag, New York, 1993).

[14] P. M. Lapostolle, IEEE Trans. Nucl. Sci. 18, 1101 (1971).

[15] F. J. Sacherer, IEEE Trans. Nucl. Sci. 18, 1105 (1971).

[16] D. T. Palmer, Ph.D. thesis, Stanford University, 1998.

[17] G.P. LeSage et al., in Advanced Accelerator Concepts: Ninth Workshop, edited by P.L. Colestock and S. Kelley, AIP Conf. Proc. No. 569 (AIP, New York, 2001).

[18] G. R. Hays et al., in Proceedings of the Conference on Lasers and Electro-optics, San Francisco, 2000 (Optical Society of America, Washington, DC, 2000), p. 293.

[19] L. Young and J. Billen, Los Alamos National Laboratory Report No. LA-UR-96-1835, 1996.

[20] M. Ferrario et al., in Proceedings of the ICFA Advanced Accelerator Workshop on the Physics of High Brightness Beams, Los Angeles, 1999 (World Scientific, Singapore, 2000).

[21] A. F. Bielajew et al., SLAC Report No. SLAC-PUB-6499, 1994.

[22] E. D. Courant, Rev. Sci. Instrum. 22, 1003 (1951).

[23] E. J. Burge and D. A. Smith, Rev. Sci. Instrum. 33, 1371 (1962). 